Neuropsychiatr (2019) · 33:212

https://doi.org/10.1007/s40211-019-00326-9

\section{neuro- psychiatrie}

vereinigt mit psychiatrie \& psychotherapie

\title{
Bericht aus dem Vorstand
}

\author{
Ursula Goedl-Fleischhacker
}

(c) Springer-Verlag GmbH Austria, ein Teil von Springer Nature 2019

\author{
Liebe Kolleginnen, liebe \\ Kollegen,
}

intensiv sind wir schon in der Vorbereitung unseres Jahreskongresses in Gmunden im April 2020. Vielen Dank für die vielen interessanten Themenvorschläge vom Programmkommittee für den Kongress, die wir bestmöglich versuchen, im Programm zu berücksichtigen. Sobald das Vorprogramm fertig gestellt ist, wird es auf unserer Homepage abrufbar sein.

In der Ärzte-Ausbildungsordnung zum Arzt/zur Ärztin für Allgemeinmedizin wurde 2015 erfreulicherweise das Fach Psychiatrie und psychotherapeutische Medizin integriert. Konkret bedeutet dies, dass ÄrztInnen in Ausbildung zur Allgemeinmedizin drei Monate an einer Psychiatrischen Abteilung ausgebildet werden. Das ist eine wesentliche Verbesserung in der Ausbildung unserer zukünftigen Allgemeinmedizinerlnnen und uns ein großes Anliegen. In Österreich sind Allgemeinmedizinerlnnen erste Ansprechpartner für PatientInnen mit psychischen Störungen. Daher ist für diese besonders wichtig, im Rah-

Prim. ${ }^{\text {a }}$ Dr. ${ }^{\text {in }}$ Ursula Goedl-Fleischhacker $(\bowtie)$ Abteilungsvorständin der Allgemein

Psychiatrischen Abteilung, Krankenanstalt Rudolfstiftung

Juchgasse 25/Haus 22, 1030 Wien,

Österreich

ursula.goedl-fleischhacker@wienkav.at men der Ausbildung, neben der Akutund Notfallmedizin, auch ausreichende Kenntnisse, Erfahrungen und Fertigkeiten in Basismedizin und fachspezifischer Medizin zu erwerben. So ist eine gute Ausbildung der Allgemeinmedizinerlnnen im psychiatrischen Fach wesentlich.

In einer Überarbeitung durch die Ärztekammer von den Rasterzeugnissen und damit Inhalten der Ausbildung für Allgemeinmedizin wurden psychiatrische Inhalte leider deutlich reduziert. Der Vorstand der ÖGPP setzt sich gemeinsam mit der Bundesfachgruppe sehr dafür ein, dass die Ausbildung unserer zukünftigen Allgemeinmedizinerlnnen nicht „abgespeckt“ wird! Keinesfalls wollen wir eine Verkürzung der Ausbildungsdauer der Allgemeinmedizinerlnnen an der Psychiatrischen Abteilung.

In Österreich gibt es derzeit mehrere Aktivitäten auf unterschiedlichen Ebenen und Bereichen, die Versorgung psychisch kranker Menschen zu verbessern. Dies unterstützen wir im Sinne des Gesundheitszieles 9, nämlich die psychosoziale Gesundheit zu fördern, selbstverständlich. Bis zum Jahr 2032 bilden die 10 Gesundheitsziele des Bundesministeriums für Gesundheit und Frauen den Handlungsrahmen für eine gesundheitsförderliche Gesamtpolitik. Für eine bestmögliche Versorgung psychisch kranker Menschen sind wir selbstverständlich mit anderen Organisationen, wie z.B. dem Berufsverband Österreichischer PsychologInnen (BÖP), gut vernetzt. In einer Pressekon- ferenz in Wien hat uns unser Präsident, Herr Univ. Prof. Dr. Johannes Wancata, gut vertreten.

Leider gibt es immer wieder unerfreuliche Artikel in Tageszeitungen, in denen sehr stigmatisierend und falsch über vermeintlich psychisch kranke Menschen berichtet wird. Eine Arbeitsgruppe erabeitet gerade Richtlinien im Umgang mit Medien, damit keine oder zumindest weniger falsche oder stigmatisierende Eindrücke vermittelt werden. Anfang des nächsten Jahres ist ein weiterer Termin zur Kommunikation mit den Medien geplant. Wir werden selbstverständlich darüber informieren.

Im Mai 2020 sind wir von der Österreichischen Ärztekammer eingeladen, eine Fortbildung „Medizin im Museum“ für AllgemeinmedizinerInnen zu gestalten. Wir freuen uns, interessante Vorträge in diesem schönen Rahmen organisieren zu dürfen.

Die Befragung zur Ausbildungsevaluierung unserer AusbildungsassistentInnen wurde mit 30.09.2019 abgeschlossen. Nun wird eifrig daran gearbeitet, die Ergebnisse auszuwerten. Auf die Präsentation der Ergebnisse freuen wir uns schon.

Eine angenehme Zeit wünsche ich Ihnen allen,

herzliche Grüße,

Ihre

Ursula Goedl-Fleischhacker

Schriftführerin 